\title{
Application of Ugi Consecutive Protocol in the Synthesis of a Peptoid Analogue of Verticilide
}

\author{
Angélica de Fátima S. Barreto ${ }^{a}$, Otilie E. Vercillo ${ }^{a}$, Ludger A. Wessjohann ${ }^{b}$ and \\ Carlos Kleber Z. Andrade ${ }^{\star a}$
}
a Laboratório de Química Metodológica e Orgânica Sintética (LaQMOS), Instituto de Química, Universidade de Brasília, CP-4478, 70910-970 Brasília-DF, Brasil and ${ }^{b}$ Department of Bioorganic Chemistry, Leibniz Institute of Plant Biochemistry, Weinberg 3, 06120 Halle (Saale), Germany. *ckleber@unb.br

Keywords: Ugi reaction, peptoids and Verticilide.

\section{INTRODUCTION}

Peptoids are non-natural molecules being capable to mimic the natural structure of peptides. These mimetics are based on oligo- $N$-substituted glycine backbones which exhibit proteolytic stability and wide range of biological activities.

Verticilide 1 is a cyclic depsipeptide that exhibits insecticidal activity and was isolated from the culture broth of Verticilium sp. FK-1033 ${ }^{1}$ (Figure 1).

In order to apply the microwave-assisted synthesis of peptoids via Ugi reactions developed by our groups ${ }^{2}$ we decided to perform the synthesis of a peptoid analogue of Verticilide (Figure 1).
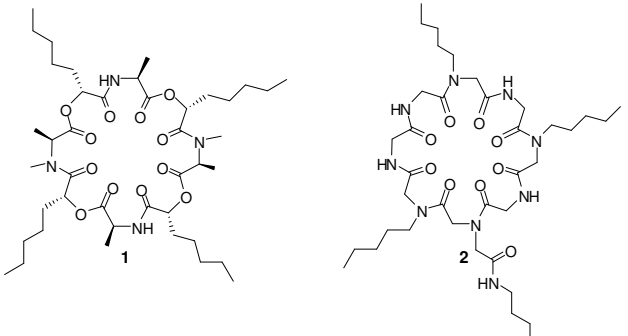

Figure 1. Verticilide (1) and its peptoid analogue (2).

\section{RESULTS AND DISCUSSION}

A convergent strategy is being used for the synthesis of 2. The retrosynthetic analysis shows that the cyclic octapeptoid 2 can be obtained by macrocyclization of acyclic substrate 3 , which can be assessed by the coupling of fragments 4 and 5 (Scheme 1).

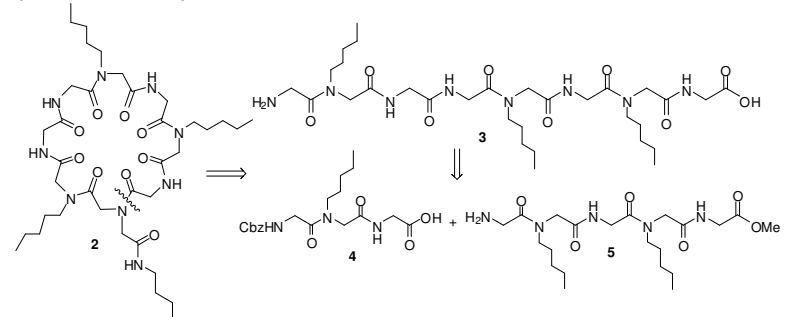

Scheme 1. Retrosynthetic analysis of cyclic octapeptoid 2.

Peptoid 4 was easily prepared by an Ugi 4component reaction (U-4CR) followed by a hydrolysis reaction (Scheme 2). Tetrapeptoid $\mathbf{5}$ was prepared using the fragment 4 in a second Ugi reaction followed by removal of the $\mathrm{Cbz}$ protecting group (Scheme 2). Both peptoids were obtained using a protocol previously established by our group. $^{2 b}$

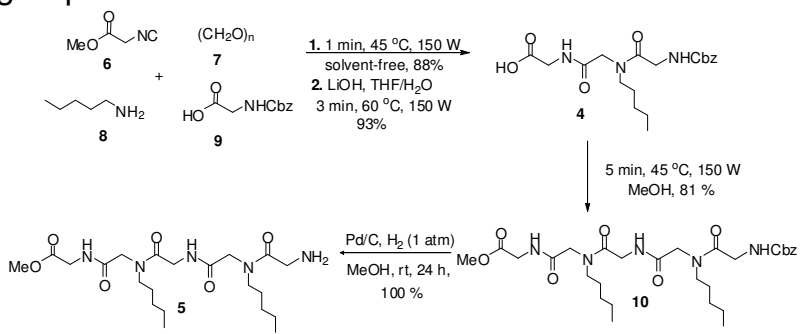

Scheme 2. Synthesis of peptoid 4 and tetrapeptoid 5.

Coupling of fragments $\mathbf{4}$ and $\mathbf{5}$ were performed using the mixed anhydride approach to give the acyclic heptapeptoid 11 in moderate yield (53\%) (Scheme 3). Subsequent ester hydrolysis and removal of the $\mathrm{Cbz}$ protecting group reactions followed by macrocyclization involving Ugi three-component four-center reaction (U-3C4CR) are in progress in our laboratory.

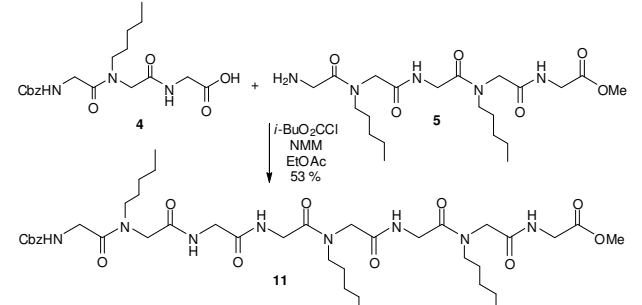

Scheme 3. Synthesis of acyclic heptapeptoid 11.

\section{CONCLUSION}

A fast and concise synthetic route allowed the synthesis of peptoid $\mathbf{4}$, tetrapeptoid $\mathbf{5}$ and acyclic heptapeptoid 11 by Ugi consecutive reactions.

\section{ACKNOWLEDGEMENTS}

IQ-UnB, Capes, CNPq and FINEP-CT INFRA no 0970/01.

\section{REFERENCES}

${ }^{1}$ Monma, S.; Sunazuka, T.; Nagai, K.; Arai, T.; Shiomi, K.; Matsui, R.; Omura, S. Org. Lett. 2006, 24, 5601.

2 (a) Vercillo, O. E.; Andrade, C. K. Z.; Wessjohann, L. A. Org. Lett. 2008, 10, 205. (b) Barreto, A .F. S.; Vercillo, O. E.; Birkett, M. A.; Caulfied, J. C.; Wessjohann, L. A.; Andrade, C. K. Z. Org. Biom. Chem. 2011 accepted. 